\title{
APPLICATION OF INTELLIGENT SWITCHING CONTROL OF PNEUMATIC ARTIFICIAL MUSCLE MANIPULATORS WITH MAGNETO-RHEOLOGICAL BRAKE
}

\author{
Diep Cong Thanh $\mathrm{TU}^{*}$ and Kyoung Kwan $\mathrm{AHN}^{* *}$ \\ * Graduate School of Mechanical and Automotive Engineering, \\ University of Ulsan, Korea \\ (E-mail: tdcthanh@yahoo.com,tdcthanh@hotmail.com) \\ ** School of Mechanical and Automotive Engineering, \\ University of Ulsan, Korea \\ San 29, Muger 2dong, Nam-gu, Ulsan, 680-764, Korea \\ (E-mail:kkahn@ulsan.ac.kr)
}

\begin{abstract}
A pneumatic artificial muscle actuator (PAM actuator), has been regarded during the recent decades as an interesting alternative to hydraulic and electric actuators because of these advantages such as high power to weight ratio, low cost, readily available and cheap power source, inherent safety and mobility assistance to humans performing tasks. However, problems with the air compressibility and the lack of damping ability have made it difficult to realize motion with high accuracy, high speed and with respect to various external inertia loads in order to realize a human-friendly therapy robot (HFTR).

An intelligent phase plane switching controller, which harmonizes a phase plane switching control (PPSC) algorithm, conventional PID controller and the adaptabilities of neural network, is newly proposed in this study. In order to realize satisfactory control performance, Magneto-Rheological Brake (MRB) is equipped to the joint of the manipulator. The experiments were carried out in practical PAM manipulator and the effectiveness of the newly proposed control algorithm was demonstrated through experiments, which proved that the stability of the manipulator could be improved greatly in a high gain control without regard to the change of external inertia loads.
\end{abstract}

\section{KEY WORDS}

Pneumatic Artificial Muscle, Magneto-Rheological Brake, Phase Plane Switching Control, Neural Network

\section{INTRODUCTION}

The PAM is undoubtedly the most promising artificial muscle for the actuation of new types of rehabilitation therapy robots. However, the air compressibility and the lack of damping ability of the PAM actuator bring the dynamic delay of the pressure response and cause the oscillatory motion. Therefore, it is not easy to realize the performance of transient response with high speed and with respect to various external inertia loads in order to realize a HFTR. The limitations of PAM manipulator have promoted research into a number of control strategies, such as an adaptive controller [2], sliding mode control [2], fuzzy PD+I learning control [3], robust control [4], feedback linearization control [5], and so on. Though these systems were successful in addressing smooth actuator motion in response to step inputs, the manipulator must be controlled slowly in order to get stable, accurate position control and the external inertia loads were also assumed to be constant or slowly varying. Therefore, intelligent control techniques have emerged to overcome some deficiencies in conventional control methods in dealing with complex real-world systems. An intelligent using a neuro-fuzzy controller [6], a hybrid using fuzzy and neural network [7] and a learning vector quantization neural network [8] were proposed. However these control algorithms were not yet to reconcile both damping and response speed in high gain control.

To overcome these problems, Electro Rheological Fluid (ERF) Damper has been applied to the PAM manipulator [9]. The results show that the ERF damper 
is one of effective methods to develop a practically available HFTR by using the PAM manipulator. However, some limitations still exist, such as the damping torque was not tuned adaptively and ERF require extremely high control voltage $(\mathrm{kV})$, works in narrow range of temperature and high nonlinearity.

Thus, the goal of this paper is to implement a MRB, to develop a fast, accurate position control system by intelligent PPSC using neural network which will tune the damping torque adaptively and without regard for the changes of external inertia loads. The experiments were carried out in practical PAM manipulator and the effectiveness of the newly proposed control algorithm with an intelligent PPSC using MRB was demonstrated through experiments.

\section{EXPERIMENTAL SETUP}

\subsection{Experimental apparatus}

The schematic diagram of the PAM manipulator is shown in Fig. 1. The hardware includes an IBM compatible PC (Pentium $1 \mathrm{GHz}$ ), which calculates the control input, controls the proportional valve (FESTO, MPYE-5-1/8HF-710 B) and MRB (LORD, MRB-21073 Rotary Brake), through D/A board (Advantech, PCI 1720), and two PAM (FESTO, MAS-10-N-220-AAMCFK). The braking torque of MRB is controlled by $\mathrm{D} / \mathrm{A}$ broad through voltage to current converter, Wonder Box Device Controller Kit (LORD, RD-3002-03). The joint angle is detected by rotary encoder (METRONIX, H40-8-3600ZO) and the air pressure into each chamber is also measured by the pressure sensors (FESTO, SDE10-10) and fed back to the computer through 24-bit digital counter board (Advantech, PCL 833) and A/D board (Advantech, PCI 1711), respectively. The external inertia load could be changed from $20\left[\mathrm{~kg} \cdot \mathrm{cm}^{2}\right]$ to $200\left[\mathrm{~kg} \cdot \mathrm{cm}^{2}\right]$, which is a $1,000[\%]$ change with respect to the minimum inertia load condition The experiments are conducted under the pressure of $0.4[\mathrm{MPa}]$ and all control software is coded in C program language. A photograph of the experimental apparatus is shown in Fig 2.

\subsection{Characteristics of MRB}

Construction of MRB was shown in Fig. 3. The rotor in Fig. 3 is fixed to the shaft, which can rotate in relation to housing. Between rotor and housing there is a gap filled with MRF. Braking torque of MRB can be controlled by the electric current in its coil. An apparent viscosity of MRF is changed at few milliseconds after the application of a magnetic field, and goes back to the normal viscosity with no magnetic field.

The following experiments are performed to investigate the characteristics of MRB, which measurement data is shown in Fig. 4. MRB is connected with a torque transducer and a servomotor in series. In this experiment, the rotational speed is changed from $100[\mathrm{rpm}]$ to $1000[\mathrm{rpm}]$ and the current applied for MRB is changed from $0[\mathrm{~A}]$ to $1[\mathrm{~A}]$. Figure 4 shows the damping torque with respect to the change of the input current (a) and rotational speed (b) of MRB. From Fig. 4 , it is clear that the damping torque of $M R B$ is independent of rotational speed and almost proportional to input current. Thus an equation (1) holds between the inputs current $I$ and damping torque $T_{b}$

$T_{b}=f(I)=a+b I$

Here, $a$ and $b$ are constant.

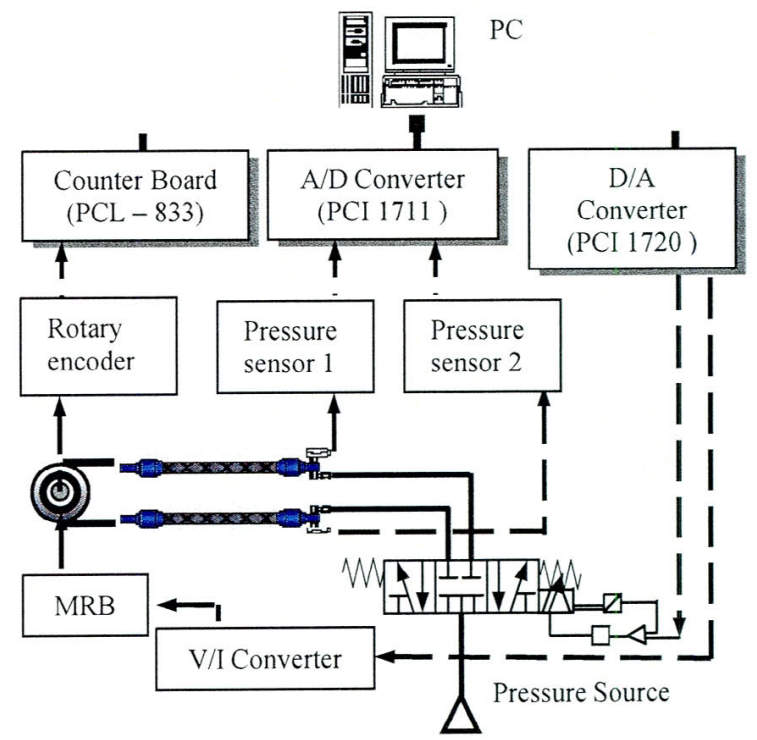

Figure 1 Schematic diagram of PAM manipulator

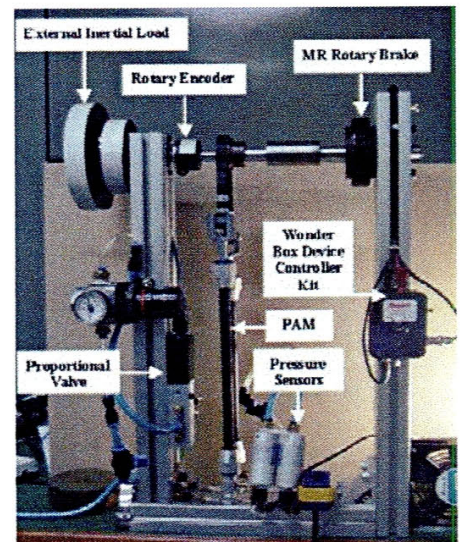

Figure 2 Photograph of the experimental apparatus

\section{CONTROL SYSTEM}

\subsection{Positioning control system}

To control this PAM manipulator, a conventional PID control algorithm was applied. The controller output at sampling sequence $k$ can be expressed as:

$$
u(k)=K_{p} e(k)+u(k-1)+\frac{K_{p} T}{T_{i}} e(k)+K_{p} T_{d} \frac{e(k)-e(k-1)}{T}
$$


where $u(k), e(k)$ are the control input to the control valve and the error between the desired set point and the output of joint, respectively.

MRB is one of effective methods because of working in only the regions where the acceleration or deceleration is too high. The structure of the PPSC algorithm is shown in Fig. 5.

Here, $s$ is Laplace operator, $T_{a}$ is torque produced by manipulator, $T_{c}$ is constant torque and $K_{E D}$ determines a gain for the torque proportional to the angular speed $\dot{\theta}$, $V_{c}$ is a control voltage of source calculated from Eq. (1) to produce $T_{c}$. A direction of a damping torque is every time opposite to the rotary direction of the arm. Eq. (3) below indicates that the damper produces a torque $T_{b}$.

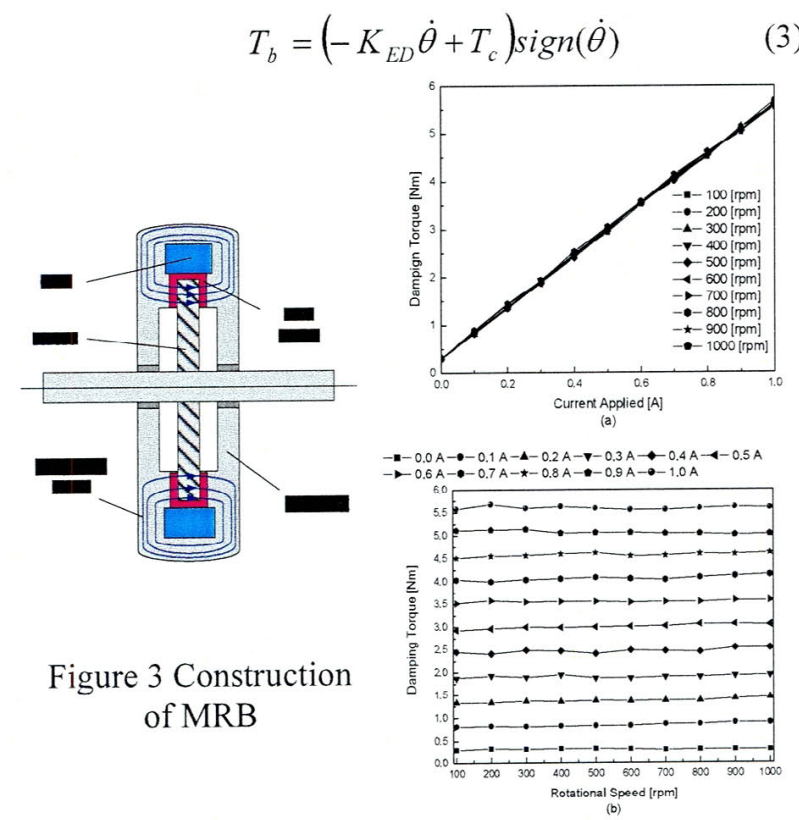

Fig. 4 Characteristics of MRB

\subsection{PPSC algorithm}

The damping torque $T_{b}$ improves the damping performance of the manipulator. In the region that the joint angle of the arm approaches to the desired angle, $a \sim b, c \sim d$ in Fig. 6(a), the current is not applied not to interfere the movement of the arm, since the high response speed is required. In the region the arm passes the desired angle, i.e. the diagonally shaded areas of $b \sim c$, $d \sim e$ in Fig. 6(a), a current is applied to improve the damping performance to converge to the desired angle quickly. To determine whether the magnetic field should be applied or not, the phase plane shown in Fig. $6(\mathrm{~b})$ is used. The horizontal axis in the phase plane corresponds to joint angle deviation $e$ between the desired angle $\theta_{r}$ and the joint angle $\theta$, and the vertical axis corresponds to the derivation of the deviation $\dot{e}=-\dot{\theta}$. Each point $a \sim e$ on the phase plane corresponds to each point $a \sim e$ in Fig. 6(a). Here, the regions with the application of current are controlled by $h$, the gradient of the line, which is shown in Fig. 6(b).
The region under the application of the damping torque expands as $|h|$ decrease.

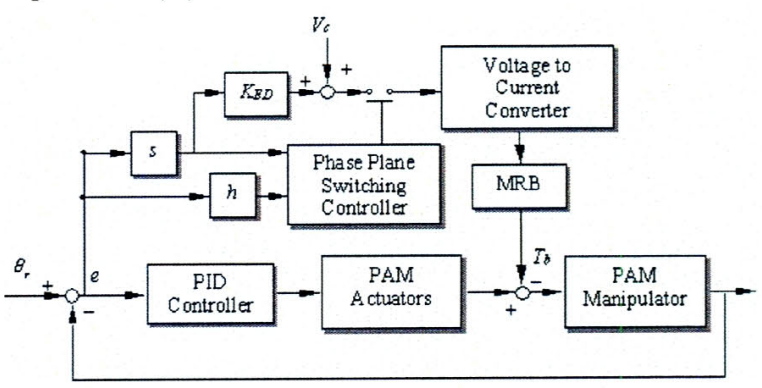

Figure 5 Block diagram of PPSC
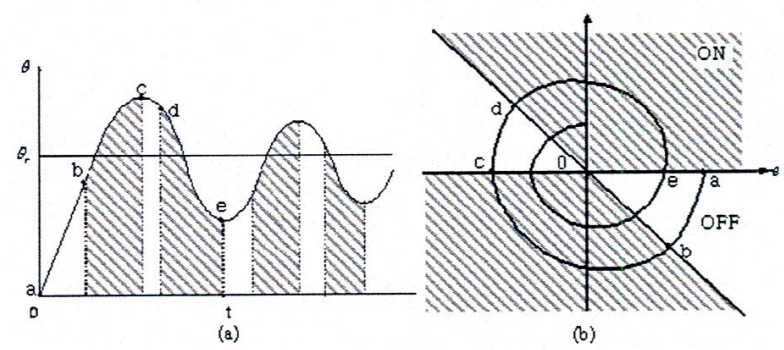

Figure 6 Concept of PPSC
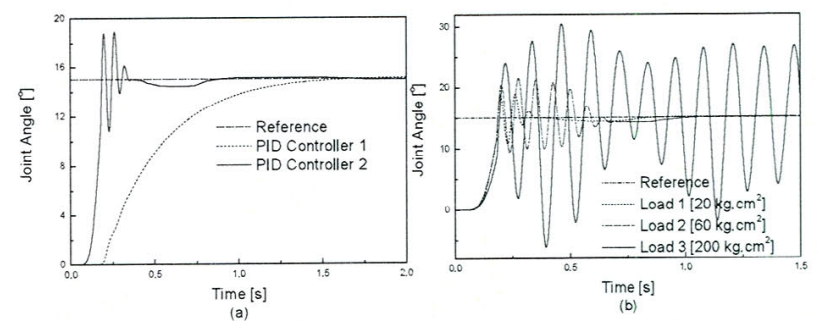

Figure 7 Experimental results of PID controller
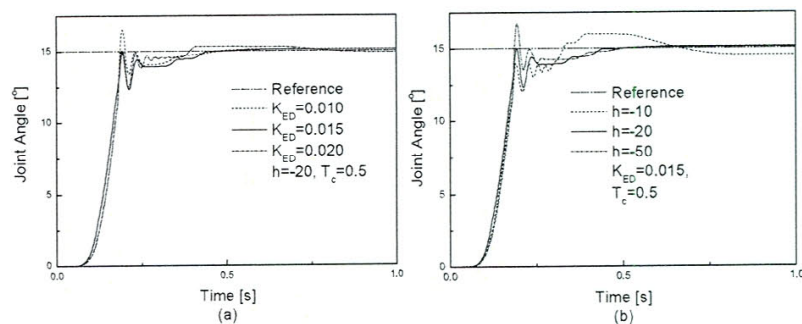

Figure 8 Experimental results of PPSC

3.3 Experimental results of PID and PPSC algorithm Experiments were carried out with 3 cases of external inertia loads $\left(20,60,200\left[\mathrm{~kg} \cdot \mathrm{cm}^{2}\right]\right)$. Figure $7(\mathrm{a})$ showed the experimental results of the PID controller where the minimum external inertia load $20\left[\mathrm{~kg} \cdot \mathrm{cm}^{2}\right]$ was used with the following 2 control parameters $K_{p}=200 \times 10^{-6} \quad K_{i}=1 \times 10^{-6} \quad, K_{d}=70 \times 10^{-6} \quad$ (PID controller 1) and $K_{p}=1000 \times 10^{-6}, K_{i}=10 \times 10^{-6}$, $K_{d}=130 \times 10^{-6}$ (PID controller 2). It is obvious that it is difficult to satisfy both the damping and response speed. The manipulator must be controlled slowly in order to have a good stability. On the contrast, the 
overshoot and oscillation are always included if one wants fast response. In addition, experimental result with PID controller 2 was shown in Fig. 7(b) where the external inertia loads change from $20\left[\mathrm{~kg} \cdot \mathrm{cm}^{2}\right]$ to 200 $\left[\mathrm{kg} \cdot \mathrm{cm}^{2}\right]$. From these results, it was understood that the system response became more oscillatory according to the increase of the external inertia load and became unstable with ten times bigger external inertia load with respect to the minimum inertia load condition.

Next, the experiments were carried out in practical PAM manipulator and the control parameters of the PPSC algorithm, were set to be $T_{c}=0.4, h=-20$ with various $K_{E D}\left(\mathrm{~K}_{\mathrm{ED}}=0.10,0.015,0.020\right)$ in experiment with load $20\left[\mathrm{~kg} \cdot \mathrm{cm}^{2}\right]$ and $K_{E D}=0.015, T_{c}=0.4$ with various $h(\mathrm{~h}=-10,-20,-50)$ in the experiment with load $60\left[\mathrm{~kg} \cdot \mathrm{cm}^{2}\right]$ in Fig. $8(\mathrm{a})$ and $8(\mathrm{~b})$, respectively. These parameters were obtained by trial-and-error through experiments. From these experimental results, it is understood that the settling time becomes very big in some value of design parameters of switching controller. To guarantee the control performance, the control parameters must be tuned adaptively and we newly proposed PPSC using neural network (PPSCNN), which is explained in detail in next section.

\section{PROPOSED PPSCNN}

\subsection{Structure of PPSCNN}

It is obvious that the PPSC algorithm is limited because the $K_{E D}$ parameter is constant. This means that the damping torque is not adaptable and optimal in any case. Here, we propose a PPSCNN, which has the adaptability of control parameter to minimize the error. With the capacity of learning and adaptability of neural network, the proposed controller can solve these problems. The damping torque will be tuned adaptively and optimally in order to minimize the position error without respect to the variation of external inertia loads. Figure 9 shows the structure of newly proposed PPSCNN. In Fig. 9, the proportional gain $K_{E D}$ was modified by neural network. The block diagram of neural network is shown in Fig. 10. Here $K_{E}(k), K_{E D}(k)$, $e(k), e_{D}(k), x(k)$ and $f(x)$ are the proportional gain, the derivative gain, the system error between desired angle output and output of joint of the PAM manipulator, the difference of the system error, control input of MRB and sigmoid function of neural network, respectively. The sigmoid function, $f(x)$, which has a nonlinear relation is presented in the following equation:

$$
f(x)=\frac{2\left(1-e^{-x \cdot Y g}\right)}{Y_{g}\left(1+e^{-x \cdot Y g}\right)}
$$

where $x$ is the input of sigmoid function and $Y_{g}$ is the parameter determining its shape. Figure 11 shows the shapes of sigmoid function with various $Y_{g}$.
We have two-layered nonlinear neurons. Neural networks are trained by the conventional back propagation algorithm to minimize the system error between the output of joint of the PAM manipulator and desired angle. In Fig. 10, the input signal of the sigmoid function in the output layer, $x(k)$, becomes:

$$
\begin{aligned}
& x(k)=K_{E}(k) e(k)+K_{E D}(k) e_{D}(k) \\
& e(k)=\theta_{r}(k)-\theta(k), \quad e_{D}(k)=\frac{e(k)\left(1-z^{-1}\right)}{\Delta T} \\
& \Delta T: \text { sampling time, } z: \text { operator of } Z-\text { transform, } \\
& k: \text { discrete sequence }
\end{aligned}
$$

$\theta_{r}(k)$ and $\theta(k)$ are desired angle and output of joint of the PAM manipulator, respectively.

The damping torque of newly proposed PPSCNN can be obtained as the following equation:

$T_{b}(k)=\left(a b s(f(x(k)))+T_{c}\right) \operatorname{sign}(\dot{\theta})$

where function $\operatorname{sign}(\dot{\theta})$ is the sign of angular speed, and function $a b s(f(x(k)))$ is the absolute value of the output of neural network.

\subsection{Learning algorithm of neural network}

In order to get the optimal value of damping torque, the control parameters $K_{E}$ and $K_{E D}$ must be adjusted automatically in order to minimize the position error. To tune $K_{E}$ and $K_{E D}$, the steepest descent method using the following equation was applied.

$$
\begin{aligned}
& K_{E}(k+1)=K_{E}(k)-\eta_{E} \frac{\partial E(k)}{\partial K_{E}} \\
& K_{E D}(k+1)=K_{E D}(k)-\eta_{E D} \frac{\partial E(k)}{\partial K_{E D}}
\end{aligned}
$$

where $\eta_{E}$ and $\eta_{E D}$ are learning rates determining convergence speed, and $E(k)$ is the error defined by the following equation:

$$
E(k)=\frac{1}{2}\left(\theta_{r}(k)-\theta(k)\right)^{2}
$$

Using the chain rule, we get the following equations:

$$
\begin{aligned}
& \frac{\partial E(k)}{\partial K_{E}}=-\frac{\partial \theta(k)}{\partial u} f^{\prime}(x(k)) e^{2}(k) \\
& \frac{\partial E(k)}{\partial K_{E D}}=-\frac{\partial \theta(k)}{\partial u} f^{\prime}(x(k)) e(k) e_{D}(k) \\
& f^{\prime}(x)=4 \frac{e^{-x \cdot Y g}}{\left(1+e^{-x \cdot Y g}\right)^{2}}
\end{aligned}
$$

As done by Yamada and Yabuta [13], for convenience, $\frac{\partial \theta(k)}{\partial u}=1$ is assumed. Eq. (8) is expressed as follows: 


$$
\begin{aligned}
& K_{E}(k+1)=K_{E}(k)+\eta_{E} e(k) e(k) \frac{4 e^{-x \cdot Y g}}{\left(1+e^{-x \cdot Y g}\right)^{2}} \\
& K_{E D}(k+1)=K_{E D}(k)+\eta_{E D} e(k) e_{D}(k) \frac{4 e^{-x \cdot Y g}}{\left(1+e^{-x \cdot Y g}\right)^{2}}
\end{aligned}
$$

From Eq. (12), it is obvious that the damping torque will be auto-tuned adaptively and optimally. However, from the experimental results, it is understood that $Y_{g}$ is difficult to determine in some cases. Thus, in case of PPSCNN, the experimental results were shown in both cases (with and without tune $Y_{g}$ ). The shape function $Y_{g}$ was auto-tuned by the following equation.

$$
\begin{aligned}
& Y_{g}(k+1)=Y_{g}(k)-\eta_{Y_{g}} \frac{\partial E(k)}{\partial Y_{g}} \\
& =Y_{g}(k)+\eta_{Y_{g}} e(k)\left(\frac{4 x(k) Y_{g}(k) e^{-x \cdot Y_{g}}+2 e^{-2 \cdot x \cdot Y_{g}}-2}{\left(1+e^{-x \cdot Y_{g}}\right)^{2}}\right)
\end{aligned}
$$

Here, $\eta_{Y_{g}}$ is learning rates determining convergence speed of shape function.

The effectiveness of the newly proposed PPSCNN will be demonstrated through experimental results with various external inertia loads.

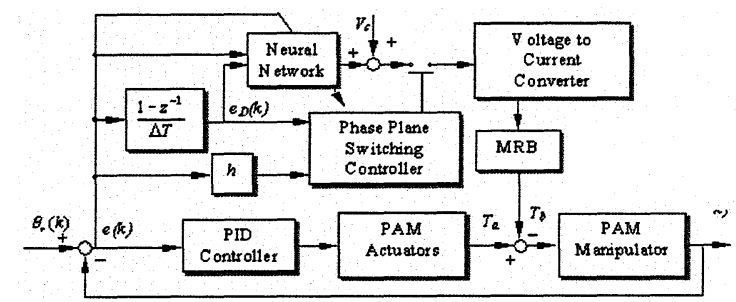

Figure 9 The structure of PPSCNN

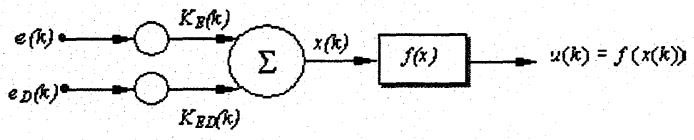

Figure 10 The block diagram of neural network

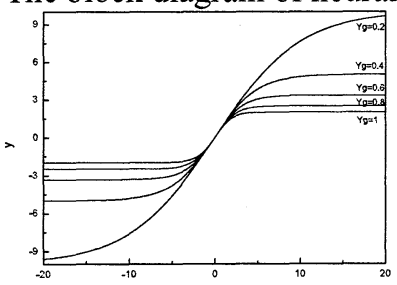

Figure 11 The sigmoid function shapes

\subsection{Experimental results}

The initial control parameters of PPSCNN were set to be $T_{c}=0.25, h=-10, K_{E}=0.010, K_{E D}=0.010 Y_{g}=1$, $\eta_{E}=350 \times 10^{-8}, \eta_{E D}=1 \times 10^{-8}$ and $\eta_{Y_{g}}=8 \times 10^{-3}$. These control parameters were obtained by trial-and-error through experiments.

Figure 12 shows the comparison between the conventional PID controller 2, the PPSC algorithm and the proposed PPSCNN with respect to various external loads. In the experiments, the joint angle of PAM manipulator was in good agreement with that of reference by using PPSC. With up to ten times bigger external inertia load with respect to the minimum external inertia load, a good control performance was also obtained in this case. However, it has too large settling time due to the fixed control parameter of PPSC. It was found that a good control performance and strong robustness were obtained without respect to the variation of external inertia load, the overshoot was almost reduced and the steady state error was reduced within $\pm 0.025^{\circ}$ by using PPSCNN. It is clear that the newly proposed controller using neural network with auto-tuning shape function was very effective in any case of external inertia loads.

The effectiveness of the newly proposed PPSCNN was shown in detail in Fig. 13 with respect to various external inertia loads. From these figures, it is understood that the damping torque was not applied for fast response when the manipulator started to move and the damping torque was generated by MRB to the rotational axis of PAM manipulator in order to reduce the overshoot and oscillation when the manipulator reaches the desired angle. In addition, during the experiments of the PAM manipulator, the control parameters $K_{E}, K_{E D}$ and the shape function of neural network, $Y_{g}$, were tuned adaptively by neural network to minimize the position error. Indeed in the experiment, it is difficult to get an optimal value of $Y_{g}$. But with newly proposed intelligent control algorithm, the shape function of neural network will be tuned adaptively and optimally in any case of load condition. And the damping torque was applied and released frequently according to the approach to the desired angle.

\section{CONCLUSION}

A novel intelligent PPSC using MRB is newly proposed and applied to the PAM manipulator in this study. In order to reconcile both damping and response speed in high gain control, a MRB had joint to the manipulator, which damping torque will be tuned adaptively and optimally by the capacity of learning and adaptability of neural network with auto-tuning shape function.

From the experimental results, it was verified that the newly proposed control algorithm presented in this study was very effective in high gain control, good control performance, fast response and strong robust stability with respect to the $1,000 \%$ change of external inertia loads and the steady state error with respect to various loads was reduced within $\pm 0.025^{\circ}$.

\section{ACKNOWLEDGEMENT}

This work was supported by Ministry of Commerce, Industry and Energy (MOCIE) of Republic of Korea, through the Research Center for Machine Parts and Materials Processing at the University of Ulsan. 


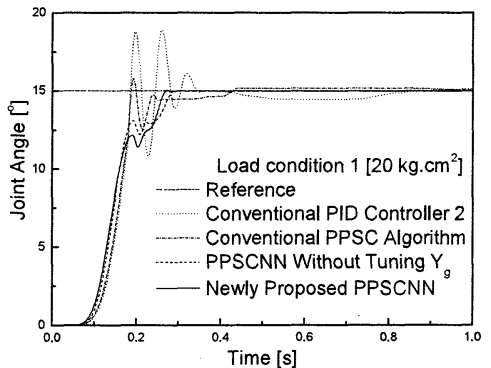

(a)
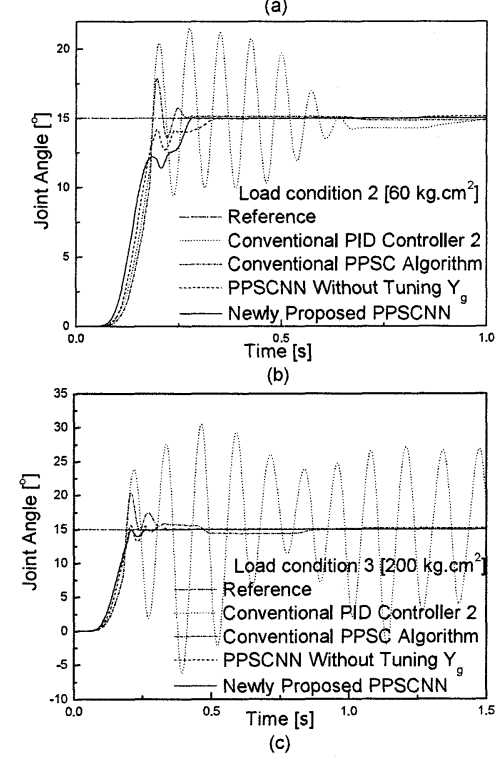

Figure 12 Comparison between PID controller 2, PPSC and PPSCNN with various external inertia loads

\section{REFERENCES}

1. Caldwell, D.G., Medrano-Cerda, G.A. and Goodwin, M.J., Control of pneumatic muscle actuators, IEEE Contr., Syst., Mag., 1995, 15-1, pp.40-48.

2. Tondu, B. and Lopex, P., Modeling and control of Mckbben artificial muscle robot actuators, IEEE Contr., Syst., Mag., 2000, 20-1, pp.15-38.

3. Chan, S.W., Lilly, J.H., Repperger, D.W. and Berlin, J.E., Fuzzy PD+I learning control for a PM, IEEE Int., Conf., Fuzzy Systems, 2003, 1, pp.278-283.

4. Guihard, M. and Gorce, P., Dynamic control of an artificial muscle arm, Proc., IEEE Int., Conf., Systems, Man and Cybernetics, 1999, 4, pp.813-818.

5. Kimura, T., Hara, S., Fujita, T. and Kagawa, T., Control for pneumatic actuator systems using feedback linearization with disturbance rejection, Proc., Conf., American Control, 1995, 1, pp.825-829.

6. Iskarous, M. and Kawamura, K., Intelligent control using a neuro-fuzzy network, Proc., IEEE Int., Conf., Intelligent Robots and Systems, 1995, 3, pp.350-355.

7. Folgheraiter, M., Gini, G., Perkowski, M. and Pivtoraiko, M., Adaptive Reflex Control for an Artificial Hand, Proc., Sym. on Robot Control, 2003.

8. Ahn, K.K. and Thanh, T.D.C., Improvement of the control performance of pneumatic artificial muscle manipulator using an intelligent switching control method, KSME, Int., Jour., 2004,8-8, pp.1388-1400.
9. Noritsugu, T., Tsuji, Y. and Ito, K., Improvement of control performance of PAM manipulator by using ER fluid damper, Proc., IEEE Int., Conf., Systems, Man and Cybernetics, 1999, 4, pp.788-793.

10. Yamada, T. and Yabuta, T., Neural network controller using autotuning method for nonlinear functions, IEEE Trans., Neural Networks, 1992, 3, pp.595-601.
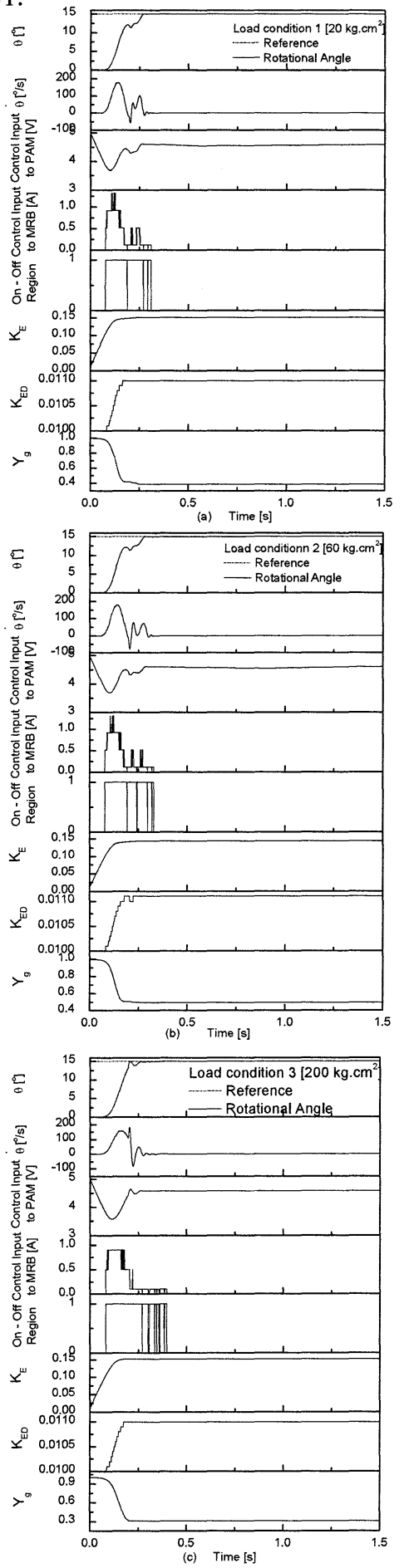

Figure 13 Experimental results of the newly proposed PPSCNN with various loads 